\title{
Application of the zero separation theorem to the restricted primitive model of electrolyte solutions
}

\author{
Sloth, Peter
}

Published in:

Journal of Chemical Physics

Link to article, DOI:

$10.1063 / 1.455651$

Publication date:

1988

Document Version

Publisher's PDF, also known as Version of record

Link back to DTU Orbit

Citation (APA):

Sloth, P. (1988). Application of the zero separation theorem to the restricted primitive model of electrolyte solutions. Journal of Chemical Physics, 89(8), 5078-5080. https://doi.org/10.1063/1.455651

\section{General rights}

Copyright and moral rights for the publications made accessible in the public portal are retained by the authors and/or other copyright owners and it is a condition of accessing publications that users recognise and abide by the legal requirements associated with these rights.

- Users may download and print one copy of any publication from the public portal for the purpose of private study or research.

- You may not further distribute the material or use it for any profit-making activity or commercial gain

- You may freely distribute the URL identifying the publication in the public portal

If you believe that this document breaches copyright please contact us providing details, and we will remove access to the work immediately and investigate your claim. 


\title{
Application of the zero separation theorem to the restricted primitive model of electrolyte solutions
}

\author{
Peter Sloth \\ Fysisk-Kemisk Institut and Center for Modellering, ikke-lineære systemers Dynamik og Irreversibel \\ Termodynamik (MIDIT), Technical University of Denmark, Bldg. 206, DK 2800 Lyngby, Denmark
}

(Received 17 May 1988; accepted 11 July 1988)

\begin{abstract}
Calculations of activity coefficients by application of the zero separation theorem is discussed for the restricted primitive model of electrolyte solutions. Numerical results within the framework of the hypernetted chain approximation are given. These indicate that the bridge functions-at zero separation-as a first approximation might be quite well represented by the corresponding function for an uncharged hard sphere system.
\end{abstract}

\section{INTRODUCTION}

In this paper the so-called zero separation theorem (ZST) is applied to the restricted primitive model (RPM) of electrolyte solutions.

In the RPM the total potential energy is given by a sum of pair potentials only. Let $g_{i j}\left(\mathrm{r}_{12}\right)$ and $u_{i j}\left(\mathrm{r}_{12}\right)$ be the radial distribution function and the pair potential function, respectively, between a particle of species $i$ at $\mathbf{r}_{1}$ and a particle of species $j$ at $\mathbf{r}_{2}$. The ZST then gives a relationship between a thermodynamic property of the system and the limit $r \rightarrow 0$ of $y_{i j}\left(\mathbf{r}_{12}\right) \equiv g_{i j}\left(\mathbf{r}_{12}\right) \exp \left[u_{i j}\left(\mathbf{r}_{12}\right) / k T\right]$. ( $T$ is the absolute temperature and $k$ Boltzman's constant.) The ZST was first worked out explicitly for the one-component hard sphere system $^{1,2}$ in the 1960's and has more recently been discussed for systems with long-range forces. ${ }^{3}$

In Sec. II the calculation of activity coefficients for the RPM - by application of the ZST-is discussed in some details within the framework of the hypernetted chain (HNC) approximation. ${ }^{4-6}$ In Sec. III numerical results-based on the HNC theory-are given, and it is found that although the HNC approximation may yield good results for $y_{i j}\left(\mathbf{r}_{12}\right)$, for large values of $r$, this is not necessarily the case for $r \approx 0$. It should be noted that a similar conclusion has been reached in the case of neutral hard sphere systems some years ago. ${ }^{7} \mathrm{We}$ find, however, that a simple approximation concerning the "bridge functions"-at $r \approx 0$-gives a marked improvement of the results.

\section{THEORY}

As a convenient starting point, we shall use a result derived by Tenne $e t a l .^{3}$ Consider an overall electroneutral system-with volume $V \longrightarrow$ of $N$ hard, charged spheres $(N / 2$ cations and $N / 2$ anions) in a "solvent" of $N_{h}$ neutral hard spheres (with the same dielectric permittivity $\epsilon$ as the surrounding media). All the species are taken to have the same diameter, $a$. Let,+- , and $h$ denote cations, anions, and neutral hard spheres, respectively. The pair potential functions $u_{i j}\left(\mathbf{r}_{12}\right)=u_{i j}(r)$ in this system are then given by

$$
\begin{aligned}
& u_{++}(r)=u_{--}(r)=u^{\mathrm{HS}}(r)+(z e)^{2} / 4 \pi \epsilon r, \\
& u_{+-}(r)=u^{\mathrm{HS}}(r)-(z e)^{2} / 4 \pi \epsilon r \\
& u_{h+}(r)=u_{h-}(r)=u_{h h}(r)=u^{\mathrm{HS}}(r) .
\end{aligned}
$$

In Eqs. (1) $e$ is the electron charge and $z$ the valence number of the electrolyte. $u^{\mathrm{HS}}(r)$ is the hard sphere pairpotential function, defined by

$$
u^{\mathrm{HS}}(r)=\left\{\begin{array}{ll}
\infty, & r<a \\
0, & a \leqslant r
\end{array} .\right.
$$

We notice that systems defined through Eqs. (1) become identical to the RPM of electrolyte solutions in the limit $\rho_{h} \equiv N_{h} / V \rightarrow 0$. According to Tenne et al.,$^{3}$ the ZST for the mixture of hard charged spheres and hard neutral spheres, specified by Eqs. (1), might be given as

$$
\ln y_{ \pm}=\ln \left[y_{+-}(0) y_{\alpha h}(0)\right]^{1 / 2} \text {. }
$$

In Eq. (3) $y_{ \pm}$is the molar mean ionic activity coefficient of the electrolyte [not to be confused with the function $y_{+-}(r)$ ], and the index $\alpha$ may be either,+- , or $h \cdot y_{i j}(r)$ can be expressed by the following exact relation ${ }^{4}$ :

$$
\begin{aligned}
& y_{i j}(r)=\exp \left[\gamma_{i j}(r)+B_{i j}(r)\right], \\
& \gamma_{i j}(r) \equiv h_{i j}(r)-c_{i j}(r),
\end{aligned}
$$

where $c_{i j}(r)$ are the direct correlation functions, $B_{i j}(r)$ the bridge functions, and $h_{i j}(r)=g_{i j}(r)-1 . c_{i j}(r)$ and $h_{i j}(r)$ are related via the Ornstein-Zernike $(\mathrm{OZ})$ equation:

$$
\gamma_{i j}\left(\mathbf{r}_{12}\right)=\sum_{k} \rho_{k} \int c_{i k}\left(\mathbf{r}_{13}\right) h_{k j}\left(\mathbf{r}_{23}\right) d \mathbf{r}_{3} \text {. }
$$

In Eq. (5) the summation is taken over all species in the system.

The HNC approximation is obtained by setting $B_{i j}(r)=0 .{ }^{4.5}$ This seems to be a reasonable approximation for $r \geqslant a$ but less so for $r<a$, as will be shown later on. Equation (4) might be rewritten as

$$
\begin{aligned}
& y_{i j}(r)=y_{i j}^{\mathrm{HS}}(r) \exp \left[\Delta \gamma_{i j}(r)+\Delta B_{i j}(r)\right], \\
& \Delta \gamma_{i j}(r) \equiv \gamma_{i j}(r)-\gamma_{i j}^{\mathrm{HS}}(r), \\
& \Delta B_{i j}(r) \equiv B_{i j}(r)-B_{i j}^{\mathrm{HS}}(r),
\end{aligned}
$$

where the HS indicates functions for the corresponding uncharged hard sphere system.

Setting $\Delta B_{i j}(r)=0$ in Eq. (6) corresponds to the reference HNC (RHNC) approximation ${ }^{8}$ - with the uncharged hard sphere system as the reference system-and this seems to be a less drastic approximation than setting $B_{i j}(r)=0$. 
From Eqs. (3) and (6) we find, using the exact relation ${ }^{1,2}$ $y^{\mathrm{HS}}(0)=y_{ \pm}^{\mathrm{HS}}$,

$$
\begin{aligned}
\ln y_{ \pm}= & \ln y_{ \pm}^{\mathrm{HS}}+(1 / 2)\left[\Delta \gamma_{+-}(0)+\Delta \gamma_{a h}(0)\right] \\
& +(1 / 2)\left[\Delta B_{+-}(0)+\Delta B_{\alpha h}(0)\right] .
\end{aligned}
$$

If the last term in Eq. (7) is neglected, we finally obtain $\ln y_{ \pm} \simeq \ln y_{ \pm}^{\mathrm{HS}}+(1 / 2)\left[\gamma_{+-}(0)+\gamma_{\alpha h}(0)\right]-\gamma^{\mathrm{HS}}(0)$.

From Eq. (5) we find in the limit $\rho_{h} \rightarrow 0(\rho \equiv N / V)$ :

$$
\begin{aligned}
\gamma_{+h}\left(\mathbf{r}_{12}\right)= & (\rho / 2) \int\left\{c_{++}\left(\mathbf{r}_{13}\right) h_{+h}\left(\mathbf{r}_{32}\right)\right. \\
& \left.+c_{+-}\left(\mathbf{r}_{13}\right) h_{-h}\left(\mathbf{r}_{32}\right)\right\} d \mathbf{r}_{3}
\end{aligned}
$$

or

$$
\gamma_{+h}\left(\mathbf{r}_{12}\right)=\rho \int c_{A}\left(\mathbf{r}_{13}\right) h_{+h}\left(\mathbf{r}_{32}\right) d \mathbf{r}_{3},
$$

where

$$
c_{A}(r) \equiv(1 / 2)\left[c_{++}(r)+c_{+-}(r)\right] .
$$

Equation (9) might be used in connection with a suitable approximative relation between $\gamma_{+h}(\mathbf{r})$ and $h_{+h}(\mathbf{r})$ to obtain $\gamma_{+h}(0)=\gamma_{\alpha h}(0)$. \{In the case of the HNC approximation we, i.e., have the following closure: $h_{+h}(r)=\exp \left[\gamma_{+h}(r)\right]-1$ for $a<r$ and $h_{+h}(r)=-1$ for $r<a$.\}

In order to apply Eq. (8) in actual calculations, we need to know the exact hard sphere quantities $\ln y_{ \pm}^{\mathrm{HS}}$ and $\gamma^{\mathrm{HS}}(0)$. The Carnahan-Starling equation of state ${ }^{9}$ provides an accurate expression for $\ln y_{ \pm}^{\mathbf{H S}}$ :

$$
\ln y_{ \pm}^{\mathrm{HS}}=\eta\left(8-9 \eta+3 \eta^{2}\right) /(1-\eta)^{3},
$$

where

$$
\eta \equiv \pi \rho a^{3} / 6 .
$$

For the calculation of $\gamma^{\mathrm{HS}}(0)$ one might-as a first approximation-use the analytical expression obtained by the Percus-Yevick (PY) theory ${ }^{10,11}$ :

$$
\gamma^{\mathrm{HS}}(0)=-\left[1+c^{\mathrm{HS}}(0)\right] \simeq(1+2 \eta)^{2} /(1-\eta)^{4}-1 .
$$

The PY theory is found to yield a good approximation to $c(r)$ for neutral hard spheres-at densities that are not too high - and we believe that formula (11) is quite accurate in the density regime which is investigated in this work. This is supported by comparison of values obtained by Eq. (11) with the data based on Monte Carlo (MC) calculations given by Groot $e t a l .^{12}$ For $\rho a^{3}=0.2$, which correspond to the
TABLE I. $\gamma_{+-}(0)$ and $\gamma_{a h}(0)$ values obtained by the HNC approximation for $B=1.681$.

\begin{tabular}{lll}
\hline \multicolumn{1}{c}{$\rho^{*}$} & $\gamma_{+-}(0)$ & $\gamma_{a h}(0)$ \\
\hline 0.0008424 & -0.1970 & 0.0035 \\
0.009595 & -0.4980 & 0.0406 \\
0.03930 & -0.6835 & 0.1750 \\
0.09248 & -0.6460 & 0.4573 \\
0.1819 & -0.2523 & 1.087 \\
\hline \hline
\end{tabular}

highest density studied in this work, Eq. (11) is found to reproduce the $\mathrm{MC}$ result with the three significant digits given in Ref. 12.

In passing, we also note that if $\gamma_{+-}(0)$ and $\gamma_{\alpha h}(0)$ results given by the mean spherical approximation ${ }^{13}$ are inserted into Eq. (8), we find

$$
\begin{aligned}
\ln y_{ \pm} \simeq & \ln y_{ \pm}^{\mathrm{HS}}-\frac{(z e)^{2}}{4 \pi \epsilon k T a} \frac{x+1-(1+2 x)^{1 / 2}}{x} \\
& +(1+2 \eta)^{2} /(1-\eta)^{4}-1-\gamma^{\mathrm{HS}}(0),
\end{aligned}
$$

where $x \equiv \kappa a, \kappa$ being the inverse Debye-Hückel length. By application of Eq. (11) the above equation reduces to the traditional MSA result as found from the energy equation.

\section{NUMERICAL RESULTS}

We have calculated $\gamma_{+-}(0)-$ and $\gamma_{+h}(0)=\gamma_{\alpha h}(0)$ values by the HNC approximation for parameters corresponding to aqueous 1:1 electrolytes. It should be pointed out that application of the HNC theory-in connection with Eq. (8) -is not inconsistent with the assumption $B_{i j}(0)$ $=B_{i j}^{\mathrm{HS}}(0)$, since $B_{i j}(r)$ is undefined for $r<a$ in the numerical solution of the $\mathrm{HNC}$ approximation.

In the RPM, an electrolyte is completely specified by two dimensionless parameters, $\rho^{*} \equiv \rho a^{3}$ and $B \equiv(z e)^{2} /$ $4 \pi \epsilon k T a$. In Table I results for $\gamma_{+-}(0)$ and $\gamma_{\alpha h}(0)$ are given for $B=1.681$ and five different concentrations in the range $0.0008424<\rho^{*}<0.1819$.

In Table II are given $\ln y_{ \pm}$results obtained by these values and Eqs. (8)-(11), as well as results from the "true" HNC expression $\left[B_{i j}(0)=0\right]$ :

$$
\ln y_{ \pm}^{\mathrm{HNC}}=(1 / 2)\left[\gamma_{+-}(0)+\gamma_{\alpha h}(0)\right]
$$

For comparison some MC data from two independent sources are also given. The data indicated $\mathbf{M C}_{\mathbf{M S}}$ are obtained from values of the Helmholtz free estimated by multistage sampling $\mathrm{MC}^{14}$ and the osmotic coefficients given in Ref. 15. Data indicated $\mathbf{M C}_{\text {test }}$ was obtained by direct $\mathbf{M C}$

TABLE II. $\ln y_{ \pm}$values obtained by different approaches for $B=1.681$.

\begin{tabular}{lcccc}
\hline \multicolumn{1}{c}{$\rho^{*}$} & HNC & Eqs. (8)-(11) & MC $_{\text {Ms }}$ & MC $_{\text {teat }}$ \\
\hline 0.0008424 & -0.0968 & -0.0968 & & \\
0.009595 & -0.229 & -0.229 & $-0.227 \pm 0.003$ & $-0.228 \pm 0.002$ \\
0.003930 & -0.254 & -0.261 & $-0.264 \pm 0.005$ & \\
0.09248 & -0.094 & -0.136 & $-0.128 \pm 0.006$ & \\
0.1819 & 0.417 & 0.224 & $0.255 \pm 0.010$ & $0.253 \pm 0.006$ \\
\hline \hline
\end{tabular}




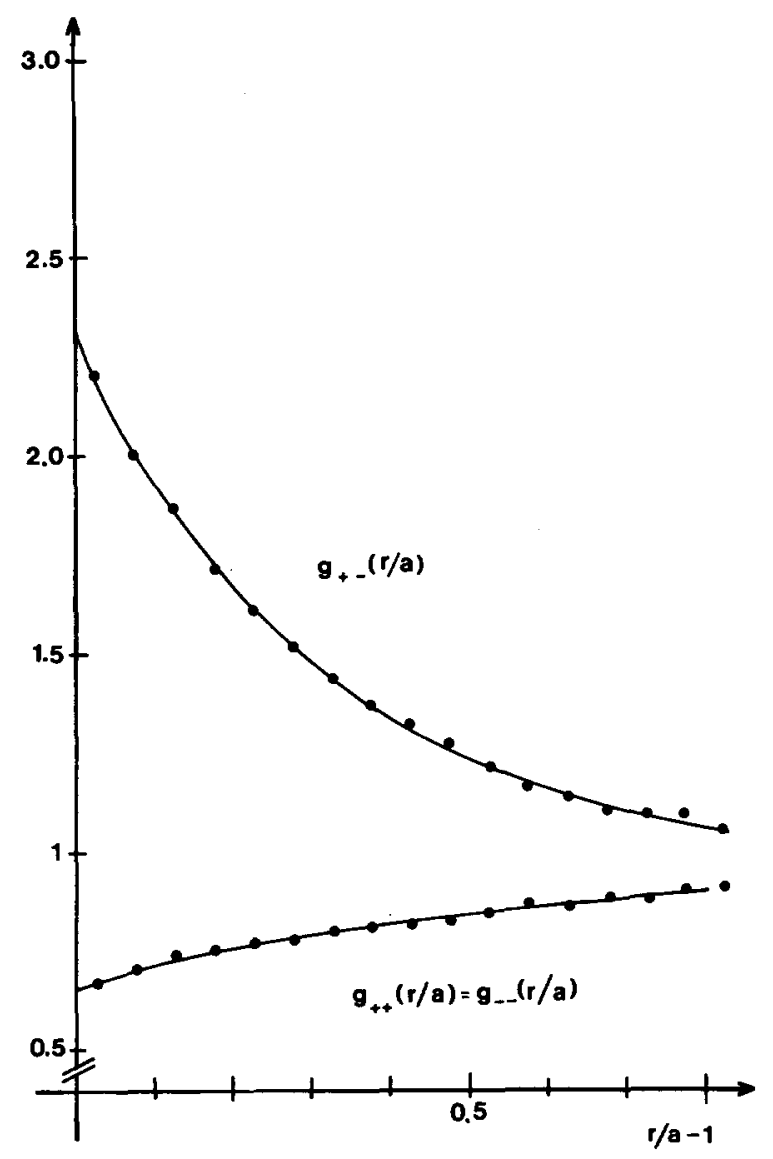

FIG. 1. Comparison of radial distribution functions obtained by the HNC approximation (full line) and a MC simulation with 256 cations and 256 anions ${ }^{17}$ (closed circles). $B=1.681, \rho^{*}=0.1819$.

simulations of the excess chemical potentials by application of the "test-particle" formula of Widom. ${ }^{16}$

From Table II it is seen, that Eqs. (8)-(11) -with application of $\mathrm{HNC}$ results for $\gamma_{i j}(0)-$ do indeed give better results for $\ln y_{ \pm}$than the $\mathrm{HNC}$ approximation itself, at the higher concentrations. At the highest concentration the HNC result is about $65 \%$ in error, whereas the assumption $B_{i j}(0)=B^{\mathrm{HS}}(0)$ gives a result which is "only" about $10 \%$ in error. This indicates that the bridge functions-at $r \approx 0$ might be quite well approximated by $B_{i j}(r) \simeq B^{\mathrm{HS}}(r)$. We also note that even though the $\mathrm{HNC}$ approximation fails to some extent at $r \approx 0$, it is a good approximation for $r \geqslant a$ (outside the hard core). This is readily seen from Fig. 1 where HNC results for the radial distribution functions, for the most dense system studied here, are compared with MC results. The agreement between the HNC and MC functions is indeed excellent!

\section{CONCLUSION}

The results obtained in this paper indicate that the bridge functions - for the RPM - might be rather well represented by the corresponding functions for a uncharged hard sphere system, at least for small values of $r$. This further suggests that the RHNC - with the neutral hard sphere system as the reference system-may yield a more consistent approximation than the HNC.

Finally we note that even though the ZST might not provide the most accurate route to $\ln y_{ \pm}$-for a given approximation-it may be used as a quite sensitive test on the validity of different kinds of approximations.

\section{ACKNOWLEDGMENTS}

I acknowledge useful discussions with Dr. T. S. Sørensen as well as financial support from Teknologistyrelsen (The National Agency for Technology) and Thomas B. Thriges Fund.

'W. G. Hoover and J. C. Poirier, J. Chem. Phys. 37, 1041 (1962).

${ }^{2}$ E. Meeron and A.J. F. Siegert, J. Chem. Phys. 48, 3139 (1968).

${ }^{3}$ R. Tenne, B. Barboy, S. Baer, and M. Silbert, Mol. Phys. 47, 913 (1982).

${ }^{4} J$. M. J. van Leeuwen, J. Groeneveld, and J. DeBoer, Physica 25, 792 (1959).

${ }^{5}$ E. Meeron, J. Math. Phys. 1, 192 (1960).

${ }^{6} J$. C. Rasaiah and H. L. Friedman, J. Chem. Phys. 48, 2742 (1968).

${ }^{7}$ D. Henderson and E.W. Grundke, J. Chem. Phys. 63, 601 (1975).

${ }^{8}$ F. Lado, Phys. Rev. A 135, 1013 (1964).

${ }^{9}$ N. F. Carnahan and K. E. Starling, J. Chem. Phys. 51, 635 (1969).

${ }^{10}$ J. K. Percus and G. J. Yevick, Phys. Rev. 100, 1 (1958).

"M. S. Wertheim, Phys. Rev. Lett. 10, 321 (1963).

${ }^{12}$ R. D. Groot, J. P. van der Eerden, and N. M. Faber, J. Chem. Phys. 87, 2263 (1987).

${ }^{13}$ E. Waisman and J. L. Lebowitz, J. Chem. Phys. 56, 3093 (1972).

${ }^{14}$ J. P. Valleau and D. N. Card, J. Chem. Phys. 57, 5457 (1972).

${ }^{15}$ J. C. Rasaiah, D. N. Card, J. P. Valleau, J. Chem. Phys. 56, 248 (1972).

${ }^{16}$ P. Sloth and T. S. Sørensen, Chem. Phys. Lett. 143, 140 (1988).

${ }^{17}$ P. Sloth (unpublished work). 